\title{
Propriedades antioxidantes do açaí (Euterpe oleracea) na síndrome metabólica
}

\author{
Antioxidant properties of acai (Euterpe oleracea) in the metabolic syndrome
}

\author{
Paula Cavalcante Amélio Silva Cedrim ${ }^{1 *}$, Elenita Marinho Albuquerque Barros', Ticiano Gomes do Nascimento \\ 1 Universidade Federal de Alagoas (UFAL), Faculdade de Nutrição (FANUT), Maceió/AL - Brasil
}

\begin{abstract}
*Corresponding Author
Paula Cavalcante Amélio Silva Cedrim, Universidade Federal de Alagoas (UFAL), Faculdade de Nutrição (FANUT), Campus A.C. Simões, Avenida Lourival de Melo Mota, s/n, km 14, CEP: 57072-970, Maceió/AL - Brasil, e-mail: ip.nutricao2@gmail.com
\end{abstract}

Cite as: Antioxidant properties of acai (Euterpe oleracea) in the metabolic syndrome. Braz. J. Food Technol., v. 21, e2017092, 2018.

Received: June 26, 2017; Accepted: Nov. 10, 2017

\section{Resumo}

Alimentos ricos em polifenóis, considerados com alto poder antioxidante, principalmente da classe antocianinas, estão sendo cada vez mais utilizados na prevenção de doenças relacionadas à síndrome metabólica. Um fruto que se destaca por apresentar essa propriedade é o açaí (Euterpe oleracea). O Brasil é o maior produtor da fruta e o mercado estrangeiro vem investindo na sua importação para utilização tanto na indústria alimentícia quanto farmacêutica. O objetivo do estudo foi realizar uma extensa revisão da literatura sobre as propriedades funcionais do açaí relacionadas à síndrome metabólica. A busca de dados foi realizada em bases, como Pubmed, Web of Science, Scielo e Science Direct. Assim, observou-se que Euterpe oleracea é um fruto rico em antocianinas, as quais atuam modulando o metabolismo lipídico para minimizar os danos no organismo causados pelo estresse oxidativo, desencadeado por doenças crônicas.

Palavras-chave: Euterpe oleracea; Síndrome metabólica; Antioxidante; Antocianinas; Flavonoides.

\section{Abstract}

Foods rich in polyphenols, considered to have high antioxidant power, mainly those in the class of anthocyanins, are increasingly being used in the prevention of diseases related to the metabolic syndrome. Acai (Euterpe oleracea) is a fruit that stands out for presenting this property, and even though Brazil is the largest producer of this fruit, the foreign market has been investing in its exportation for use in both the food and pharmaceutical industries. The objective of this study was to carry out an extensive review of the literature on the functional properties of the acai berry as related to the metabolic syndrome. The data search was carried out in databases such as Pubmed, Web of Science, Scielo and Science Direct. Thus it was observed that Euterpe oleracea is a fruit rich in anthocyanins, which act by modulating the lipid metabolism to overcome the damage caused in the body by the oxidative stress unleashed by chronic diseases.

Keywords: Euterpe oleracea; Metabolic syndrome; Antioxidant; Anthocyanidin; Flavonoids.

\section{Introdução}

Nos últimos anos, a ciência da nutrição tem alcançado o reconhecimento mundial por enfatizar a importância da alimentação saudável na promoção da saúde das populações. Além de fornecer subsídios para o planejamento da alimentação e a utilização adequada do alimento como promotor da saúde, tende a reduzir o risco de agravos e buscar a melhoria do estado de saúde (REIS et al., 2011).
Um dos agravos que vêm atingindo a população é a obesidade, que pode desencadear diabetes, doenças cardiovasculares, dislipidemia, esteatose hepática. Observe-se que a combinação de pelo menos três desses problemas é classificada como síndrome metabólica (SM) (ABETE et al., 2011).

Muitos países utilizam estratégias preventivas com informações nutricionais para poupar ou prevenir o surgimento 
da SM, visto que esses problemas de saúde desencadeiam efeitos adversos e essas terapias preventivas vêm mostrando um resultado positivo (DEVALARAJA et al., 2011).

Estudos mostram que o consumo de alimentos ricos em polifenóis, principalmente os da classe dos flavonoides, tem sido relacionado com o baixo risco no desenvolvimento de diversas doenças, devido às propriedades antioxidantes presentes no alimento. Nota-se que um fruto que vem ganhando destaque, entre esses alimentos, é o açaí (Euterpe oleracea Mart.), por apresentar em sua composição quantidades significativas de um grupo dos flavonoides, conhecido como antocianinas (LIMA et al., 2012; LIN et al., 2007).

Atualmente, alguns estudos vêm mostrando a ingestão do açaí no controle ou na prevenção de algumas doenças, como nos casos de diabetes, dislipidemia e doença cardiovascular, pois, além de o fruto demonstrar alta capacidade antioxidante, apresenta também benefícios nutricionais e terapêuticos, como, por exemplo, ação antiproliferativa, anti-inflamatória, e efeito cardioprotetor (BONOMO et al., 2014; OLIVEIRA et al., 2010).

Assim, o objetivo do estudo foi realizar uma extensa revisão da literatura sobre as propriedades funcionais do açaí relacionadas à síndrome metabólica. A busca de dados realizou-se nas seguintes bases de dados: Pubmed, Web of Science, Scielo e Science Direct, com os seguintes descritores a) "açaí or euterpe oleracea"; b) "açaí or euterpe oleracea and metabolic syndrome"; c) "açaí or euterpe oleracea and diabetes"; d) "açaí or euterpe oleracea and hipertension"; e) "açaí or euterpe oleracea and dyslipidemia"; f) "açaí or euterpe oleracea and obesity"; g) "açaí or euterpe oleracea and antioxidant"; h) "açaí or euterpe oleracea and anthocyanidin"; i) "açaí or euterpe oleracea and tocopherols"; j) "açaí or euterpe oleracea and flavonoids". A busca de dados foi limitada à língua inglesa e os artigos selecionados para análise foram aqueles que apresentaram grande pertinência ao assunto, nos anos de 2004 a 2017.

\subsection{Açaí (Euterpe oleracea)}

O açaizeiro (Euterpe oleracea Mart.), de origem nativa da América Central e do Sul, é considerado como a palmeira mais produtiva da região amazônica. Destaca-se por ser fruto exótico e é consumido como um alimento básico nessa região do Brasil. Nos últimos anos, o fruto açaí ganhou a atenção internacional como um alimento funcional, devido aos seus benefícios nutricionais e terapêuticos relacionados a alta capacidade antioxidante e sua composição fitoquímica (BONOMO et al., 2014; MENEZES et al., 2008; BERNAUD; FUNCHAL, 2011).

O fruto do açaizeiro tem uma forma globosa, de 1 a 2 cm de diâmetro, e peso médio de 1,5 grama; dependendo do tipo e da maturação, o epicarpo é roxo ou verde, porém seu consumo não ocorre na forma in natura, necessitando ser processado (ROGEZ et al., 2011).

O Brasil é considerado o país que mais produz, consome e exporta o açaí, e as indústrias, visando a manutenção e ampliação do mercado do fruto, estão investindo não só no cultivo, mas também na qualificação da mão de obra. Com o crescimento do mercado, esse produto passou a ser consumido também nas grandes capitais brasileiras, atingindo até alguns países, como Japão, China, Estados Unidos e Europa (MENEZES et al., 2008).

Existem diversas formas de apreciação do açaí, como, por exemplo, nas formas de geleias, licor, polpa congelada, xarope, pó, creme e sorvetes. No mercado brasileiro, o fruto é consumido com finalidade de bebida energética; já no mercado externo, como uma bebida exótica (DARNET et al., 2011; YAMAGUCHI et al., 2015).

\subsection{Composição química do açaí}

O açaí, além de apresentar um sabor delicioso e refrescante, é uma fruta com valor nutricional altamente energético, contendo alto teor de lipídios, carboidratos, proteínas, tornando-o um alimento calórico. Ainda apresenta, em sua composição, compostos bioativos, como polifenóis, da classe dos flavonoides, em destaque as antocianinas (DARNET et al., 2011; KANG et al., 2010).

Os flavonoides são uma grande classe de compostos polifenólicos de baixa massa molecular, que possuem um esqueleto de 15 carbonos constituído por dois anéis de benzeno, ligados através de um anel de pirano heterocíclico. Essa classe de polifenóis foi classificado em seis subgrupos: flavonas, flavonóis, flavanonas, flavan-3-ols, isoflavonas e antocianidinas (GOMES et al., 2016). No açaí, a classe das antocianinas desperta maior interesse devido à sua prevalência (POZO-INSFRAN et al., 2004).

Devido ao fruto apresentar propriedades físico-químicas com poder antioxidante, que atuam inibindo ou diminuindo os efeitos desencadeados pelos radicais livres, estudos indicam a possibilidade de o açaí fazer parte dos grupos dos alimentos funcionais (POZO-INSFRAN et al., 2004; ROCHA et al., 2015).

Antioxidantes são compostos que atuam inibindo e/ou diminuindo os efeitos do estresse oxidativo, desencadeados pelos radicais livres e compostos oxidantes. São importantes porque, com o combate aos processos oxidativos, há menores danos ao DNA e às macromoléculas, amenizando, assim, os danos cumulativos que podem desencadear doenças (SCHAUSS et al., 2006).

O alto teor dos polifenóis no açaí torna-o uma das cinco frutas com maior potencial antioxidante, apresentando diversas propriedades - anti-inflamatória e farmacológica - associadas ao combate de doenças desencadeadas por espécies reativas de oxigênio (radicas 
livres) (KANG et al., 2010; POZO-INSFRAN et al., 2006; PACHECO-PALENCIA et al., 2008a).

Análise cromatográfica da polpa de açaí mostrou a presença de uma quantidade significativa dos polifenóis, em que duas antocianinas, cianidina 3-glucosídeo e cianidina 3-rutinosídeo, foram encontradas, sendo as mais predominantes (MOURA \& RESENDE, 2016; PACHECO-PALENCIA et al., 2009). Em outro estudo, foi possível identificar seis antocianinas (Figura 1) em amostra do fruto liofilizada, entre as quais encontram-se: cianidina 3-glucosídeos; cianidina 3-rutinosídeo; cianidina-3-sambubiosídeo; peonidina-3-rutinosídeo; pelargonidina-3- glucosídeos, e delfinidina-3-glucosídeos (CARDOSO et al., 2015).

Cesar et al. (2014) relataram que o açaí apresentou maior poder antioxidante do que outros frutos ricos em antocianina, como mirtilos e amoras, e observaram que a cianidina-3-glucosídeo (1040 mg/L de polpa) era a antocianina em maior quantidade na polpa do fruto.

A Tabela 1 apresenta estudos com determinação do teor de antocianinas presentes na polpa do açaí e houve diferença na quantidade desses flavonoides, encontrados em menor quantidade no estudo de Coutinho et al. (2017), e em maior quantidade por Pacheco-Palencia et al. (2009).

Devido aos diferentes procedimentos utilizados para o preparo da polpa, como, por exemplo, a água adicionada ao produto, o teor de fitoquímicos na polpa de fruto pode variar significativamente; além disso, as condições climáticas, a variedade, a colheita e a fase de amadurecimento também podem influenciar no conteúdo dos fitoquímicos em alimentos (GORDON et al., 2012).

\subsection{Síndrome Metabólica associada ao açaí}

A SM é um progressivo estado fisiopatológico associado ao desenvolvimento de diabetes tipo 2 e doença cardiovascular aterosclerótica, entre outras doenças relacionadas ao estresse oxidativo.

O crescente interesse dos consumidores por produtos mais saudáveis, como frutas e vegetais, reflete as evidências de vários estudos científicos, que apontam o elevado consumo destes alimentos com efeitos benéficos à saúde, dentre os quais: prevenção de doenças crônicas relacionadas ao estresse oxidativo; prevenção de doenças neurodegenerativas, câncer, obesidade e doenças cardiovasculares (MASELLA et al., 2012; DEVALARAJA et al., 2011; KIM et al., 2012).

Nos últimos anos, muitas pesquisas sobre o efeito positivo do consumo de frutas de baga na saúde humana

Tabela 1. Teor de antocianinas encontrado em polpa de açaí em diversos estudos (Euterpe oleracea).

\begin{tabular}{cc}
\hline Autores & Teor de Antocianinas \\
\hline Coutinho et al. (2017) & 12,05 a $24,98 \mathrm{mg} / 100 \mathrm{~g}$ \\
Fernandes et al. (2016) & $36,38 \mathrm{mg} / 100 \mathrm{~g}$ \\
Silva et al. (2016) & 62,58 a $135,15 \mathrm{mg} / 100 \mathrm{~g}$ \\
Rufino et al. (2010) & $111 \mathrm{mg} / 100 \mathrm{~g}$ \\
Rogez et al. (2011) & 109 a $165 \mathrm{mg} / 100 \mathrm{~g}$ \\
Pacheco-Palencia et al. (2009) & $205,6 \mathrm{mg} / 100 \mathrm{~g}$ \\
\hline
\end{tabular}

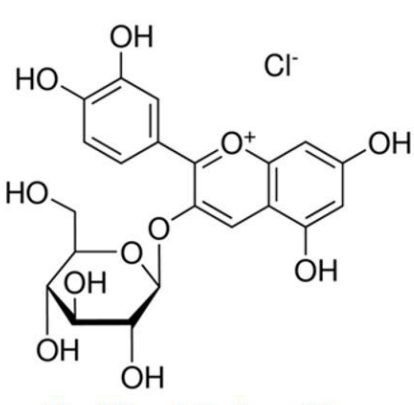

Cianidina-3-O-glucosídeo<smiles>COc1cc(-c2[o+]c3cc(O)cc(O)c3cc2OC2OC(CO)C3OC2C(O)C3O)ccc1O</smiles>

Peonidina-3-O-rutinosídeo

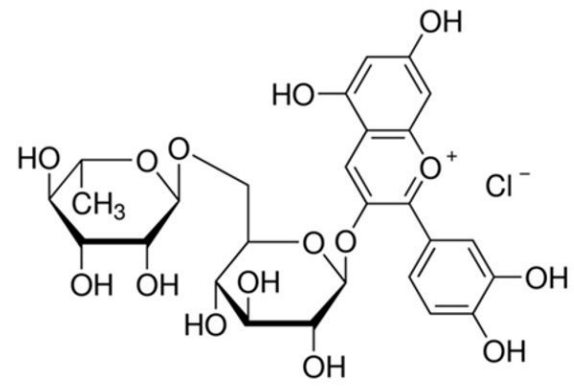

Cianidina-3-O-rutenosídeo<smiles>OCC1OC(COc2cc3c(O)cc(O)cc3[o+]c2-c2ccc(O)cc2)C(O)C(O)C1O</smiles>

Pelargonidina-3-O-glucosídeo

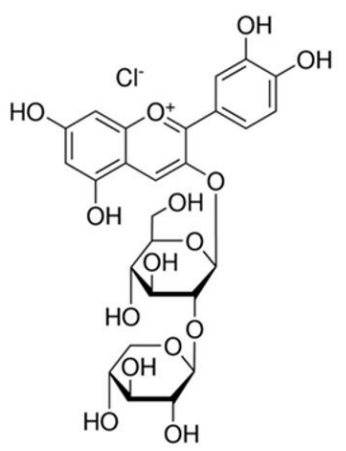

Cianidina-3-O-sambubiosídeo

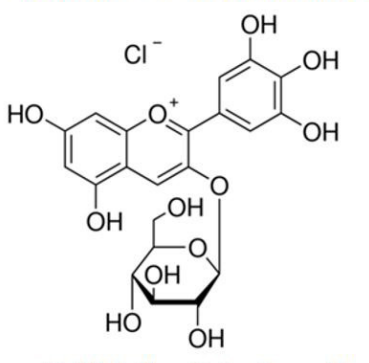

Delfinidina-3-O-glucoside

Figura 1. Estrutura química das antocianinas encontradas no açaí (Euterpe oleracea). 
e no desempenho preventivo de algumas doenças têm-se centrado nas frutas vermelhas-pretas tropicais exóticas. Destaca-se que estas bagas são fontes naturais de compostos bioativos, principalmente antocianinas e fenólicos, com características antioxidantes e anti-inflamatórias altamente promissoras (GARZÓN et al., 2017).

Efeitos benéficos do açaí têm sido demonstrados em modelos experimentais para prevenção e controle da SM, em um estudo com adultos com sobrepeso; observou-se que a suplementação de $200 \mathrm{~g}$ de polpa de açaí por dia, em indivíduos com sobrepeso, após um mês de administração, levou à diminuição nos níveis médios de glicemia em jejum, colesterol total e colesterol LDL (UDANI et al., 2011).

Um estudo com ratos que apresentavam diabetes tipo 2 demonstrou que o tratamento oral com extrato da semente do açaí conseguiu reverter o aumento dos níveis de glicemia e insulina, em receptores de insulina no tecido adiposo (OLIVEIRA et al., 2015).

Souza et al. (2010), em seu estudo, administraram polpa de açaí em ratos fêmeas Fischer, alimentados com dieta hipercolesterolêmica, e observaram que houve uma melhora na eficiência alimentar e na redução dos níveis do colesterol, lipoproteína total e não alta densidade, sugerindo um efeito hipocolesterolêmico dos produtos finais do estresse oxidativo.

O impacto das propriedades do açaí foi observado em um estudo em que houve redução do colesterol total, lipoproteína de baixa densidade (colesterol-LDL) e triacilgliceróis no sangue, sem alterar o colesterol HDL (lipoproteína de alta densidade) e VLDL (lipoproteína de baixa densidade) (YUYAMA et al., 2011).

O efeito anti-hipertensivo de açaí foi observado em estudos randomizado e duplo-cego, e controlado por placebo, com suplementação de açaí, os quais demonstraram uma redução significativa na pressão arterial sistólica (GALE et al., 2014; ZAPATA-SUDO et al., 2014).

Cordeiro et al. (2018) observaram que o extrato de sementes de açaí, rico em polifenóis, foi benéfico para a preservação da morfologia e função renal em ratos diabéticos hipertensos, tendo como efeitos benéficos a redução da pressão arterial sistólica, a melhoria da barreira de filtração renal e as ações anti-inflamatórias, antioxidantes e vasodilatadoras.

O extrato de semente de açaí, no tratamento doenças crônicas, previne o desenvolvimento de hipertensão, disfunção endotelial e alterações estruturais vasculares, por apresentar um potente efeito vasodilatador dependente do endotélio e da ação antioxidante e anti-hipertensiva (ROCHA et al., 2008; COSTA et al., 2012).

Estudos in vitro e in vivo demonstraram que a polpa pode proteger os eritrócitos humanos e as células polimorfonucleares contra o estresse oxidativo (HONZEL et al.,
2008; JENSEN et al., 2008). Esta baga também mostrou possuir atividade anticancerígena in vitro, em que extratos fenólicos de açaí exibiram a capacidade de reduzir a proliferação de leucemia e de células de adenocarcinoma de cólon humano (MERTENS-TALCOTT et al., 2008; PACHECO-PALENCIA et al., 2008b).

Os benefícios para a saúde do consumo de polpa de açaí foram demonstrados em humanos, tendo sido administrado um suco rico em polpa de açaí, em que a polpa não tinha sido clarificada ou filtrada. O estudo relatou reduções significativas na peroxidação lipídica durante o estresse oxidativo, bem como um rápido aumento da atividade antioxidante no soro, aumentando a proteção celular de espécies reativas de oxigênio, medida pela proteção antioxidante celular em eritrócitos (JENSEN et al., 2008).

\section{Conclusão}

Diante do exposto, observa-se que Euterpe oleracea é um fruto rico em antocianinas, que atuam modulando o metabolismo lipídico para melhora dos danos no organismo causados pelo estresse oxidativo, desencadeado por doenças crônicas, além de apresentar efeitos satisfatórios nos níveis de glicemia e pressão arterial, prevenindo e controlando efeitos da síndrome metabólica.

\section{Agradecimentos}

À Fundação de Amparo a Pesquisa do Estado de Alagoas (FAPEAL), ao FINEP, ao CNPq e à Coordenação de Aperfeiçoamento de Pessoal de Nível Superior (CAPES).

\section{Referências}

ABETE, I.; GOYENECHEA, E.; ZULET, M. A.; MARTINEZ, J. A. Obesity and metabolic syndrome: potential benefit from specific nutritional components. Nutrition, Metabolism, and Cardiovascular Diseases, v. 21, p. B1-B15, 2011. Suplemento 2. http://dx.doi. org/10.1016/j.numecd.2011.05.001. PMid:21764273.

BERNAUD, R. F. S.; FUNCHAL, C. D. S. Atividade antioxidante do açaí. Nutrição Brasil, v. 10, n. 5, p. 310-316, 2011.

BONOMO, L. F.; SILVA, D. N.; BOASQUIVIS, P. F.; PAIVA, F. A.; GUERRA, J. F.; MARTINS, T. A.; TORRES, Á. G. J.; PAULA, I. T.; CANESCHI, W. L.; JACOLOT, P.; GROSSIN, N.; TESSIER, F. J.; BOULANGER, E.; SILVA, M. E.; PEDROSA, M. L.; OLIVEIRA, R. P. Açaí (Euterpe oleracea Mart.) modulates oxidative stress resistance in Caenorhabditis elegans by direct and indirect mechanisms. PLoS One, v. 9, n. 3, p. e89933, 2014. http://dx.doi. org/10.1371/journal. pone.0089933. PMid:24594796.

CARDOSO, L. M.; NOVAES, R. D.; CASTRO, C. A.; NOVELLO, A. A.; GONÇALVES, R. V.; RICCI-SILVA, M. E.; RAMOS, H. J. O.; ELUZIO, M. C. G.; LEITE, J. P. V. Chemical composition, characterization of anthocyanins and antioxidant potential of 
Euterpe edulis fruits: applicability on genetic dyslipidemia and hepatic steatosis in mice. Nutrición Hospitalaria, v. 32, n. 2 , p. 702-709, 2015. http://dx. doi.org/10.3305/nh.2015.32.2.8885. PMid:26268102

CESAR, L. T.; CABRAL, M. F.; MAIA, G. A.; FIGUEIREDO, R. W.; MIRANDA, M. R.; SOUSA, P. H.; BRASIL, I. M.; GOMES, C. $L$. Effects of clarification on physicochemical characteristics, antioxidante capacity and quality atributes of açaí (Euterpe oleracea Mart.) juice. Journal of Food Science and Technology, v. 51, n. 11, p. 3293-3300, 2014. http://dx.doi.org/10.1007/ s13197-012-0809-6. PMid:26396323.

CORDEIRO, V. S. C.; BEM, G. F.; COSTA, C. A.; SANTOS, I. B.; CARVAlho, L. C. R. M.; OGNiBene, D. T.; ROCHA, A. P. M.; CARVALHO, J. J.; MOURA, R. S.; RESENDE, A. C. Euterpe oleracea Mart. seed extract protects against renal injury in diabetic and spontaneously hypertensive rats: role of inflammation and oxidative stress. European Journal of Nutrition, v. 57, n. 2, p. 817-832, 2018. PMid:28105508.

COSTA, C. A.; OLIVEIRA, P. R.; BEM, G. F.; CAVALHO, L. C.; OGNIBENE, D. T.; SILVA, A. F.; VALENÇA, S. S.; PIRES, K. M.; SOUSA, P. J. C.; MOURA, R. S.; RESENDE, A. C. Euterpe oleracea Mart.-derived polyphenols prevent endotelial dysfunction and vascular structural changes in renovascular hypertensive rats: role of oxidative stress. Naunyn-Schmiedeberg's Archives of Pharmacology, v. 385, n. 12, p. 1199-1209, 2012. http://dx.doi. org/10.1007/s00210-012-0798-z. PMid:23052352.

COUTINHO, R. M. P.; FONTES, E. A. F.; VIEIRA, L. M.; BARROS, F. A. R. D.; CARVALHO, A. F. D.; STRINGHETA, P. C. Physicochemical and microbiological characterization and antioxidant capacity of açaí pulps marketed in the states of Minas Gerais and Pará, Brazil. Ciência Rural, v. 47, n. 1, e20151172, 2017. http://dx.doi. org/10.1590/0103-8478cr20151172.

DARNET, S.; SERRA, J. L.; RODRIGUES, A. M. C.; SILVA, L. H. M. A high-performance liquid chromatography method to measure tocopherols in assai pulp (Euterpe oleracea). Food Research International, v. 44, n. 7, p. 2107-2111, 2011. http:// dx.doi.org/10.1016/j.foodres.2010.12.039.

DEVALARAJA, S.; JAIN, S.; YADAV, H. Exotic fruits as therapeutic complements for diabetes, obesity and metabolic syndrome. Food Research International, v. 44, n. 7, p. 1856-1865, 2011. http://dx.doi.org/10.1016/j.foodres.2011.04.008. PMid:21857774.

FERNANDES, E. T. M. B.; MACIEL, V. T.; SOUZA, M. L. D.; FURTADO, C. D. M.; WADT, L. H. D. O.; CUNHA, C. R. D. Physicochemical composition, color and sensory acceptance of low-fat cupuaçu and açaí nectar: characterization and changes during storage. Food Science and Technology, v. 36, n. 3, p. 413-420, 2016. http://dx.doi.org/10.1590/1678-457X.03415.

GALE, A. M.; KAUR, R.; BAKER, W. L. Hemodynamic and electrocardiographic effects of acai berry in healthy volunteers: a randomized controlled trial. International Journal of Cardiology, v. 174, n. 2, p. 421-423, 2014. http://dx.doi.org/10.1016/j. ijcard.2014.04.036. PMid:24767759.

GARZÓN, G. A.; NARVÁEZ-CUENCA, C. E.; VINCKEN, J. P.; GRUPPEN, H. Polyphenolic composition and antioxidant activity of açai (Euterpe oleracea Mart.) from Colombia. Food Chemistry, v. 217, p. 364-372, 2017. http://dx.doi.org/10.1016/j. foodchem.2016.08.107. PMid:27664647.

GOMES, S. M.; GHICA, M. E.; RODRIGUES, I. A.; GIL, E. S.; OLIVEIRA-BRETT, A. M. Flavonoids electrochemical detection in fruit extracts and total antioxidant capacity evaluation. Talanta, v. 154, p. 284-291, 2016. http://dx.doi.org/10.1016/j. talanta.2016.03.083. PMid:27154676.

GORDON, A.; CRUZ, A. P.; CABRAL, L. M.; FREITAS, S. C.; TAXI, C. M.; DONANGELO, C. M.; MATTIETTO, R. A.; FRIEDRICH, M.; MATTA, V. M.; MARX, F. Chemical characterization and evaluation of antioxidant properties of açaí fruits (Euterpe oleraceae Mart.) during ripening. Food Chemistry, v. 133, n. 2, p. 256-263, 2012. http://dx.doi.org/10.1016/j.foodchem.2011.11.150. PMid:25683393.

HONZEL, D.; CARTER, S. G.; REDMAN, K. A.; SCHAUSS, A. G.; ENDRES, J. R.; JENSEN, G. S. Comparison of chemical and cellbased antioxidant methods for evaluation of foods and natural products: generating multifaceted data by parallel testing using erythrocytes and polymorphonuclear cells. Journal of Agricultural and Food Chemistry, v. 56, n. 18, p. 8319-8325, 2008. http:// dx.doi.org/10.1021/jf800401d. PMid:18717566.

JENSEN, G. S.; WU, X.; PATTERSON, K. M.; BARNES, J.; CARTER, S. G.; SCHERWITZ, L.; BEAMAN, R.; ENDRES, J. R.; SCHAUSS, A. G. In vitro and in vivo antioxidant and antiinflammatory capacities of an antioxidant-rich fruit and berry juice blend: results of a pilot and randomized, double-blinded, placebo-controlled, crossover study. Journal of Agricultural and Food Chemistry, v. 56, n. 18, p. 8326-8333, 2008. http:// dx.doi.org/10.1021/jf8016157. PMid:18717569.

KANG, J.; LI, Z.; WU, T.; JENSEN, G. S.; SCHAUSS, A. G.; WU, X. Anti-oxidant capacities of flavonoid compounds isolated from acai pulp (Euterpe oleracea Mart.). Food Chemistry, v. 122, n. 3, p. 610-617, 2010. http://dx.doi.org/10.1016/j.foodchem.2010.03.020.

KIM, J. Y.; HONG, J. H.; JUNG, H. K.; JEONG, Y. S.; CHO, K. H. Grape skin and loquat leaf extracts and acai puree have potent anti-atherosclerotic and anti-diabetic activity in vitro and in vivo in hypercholesterolemic zebrafish. International Journal of Molecular Medicine, v. 30, n. 3, p. 606-614, 2012. http://dx. doi. org/10.3892/ijmm.2012.1045. PMid:22751734.

LIMA, C. P.; CUNICO, M. M.; MIYAZAKI, C. M. S.; MIGUEL, O. G.; CÔCCO, L. C.; YAMAMOTO, C. I.; MIGUEL, M. D. Conteúdo polifenólico e atividade antioxidante dos frutos da palmeira Juçara (Euterpe edulis Martius). Revista Brasileira de Plantas Medicinais, v. 14, n. 2, p. 321-326, 2012. http://dx. doi.org/10.1590/ S1516-05722012000200011.

LIN, J.; REXRODE, K. M.; HU, F.; ALBERT, C. M.; CHAE, C. U.; RIMM, E. B.; STAMPFER, M. J.; MANSON, J. E. Dietary intakes of 
Propriedades antioxidantes do açaí (Euterpe oleracea) na síndrome metabólica

Cedrim, P. C. A. S. et al.

flavonols and flavones and coronary heart disease in US women. American Journal of Epidemiology, v. 165, n. 11, p. 1305-1313, 2007. http://dx.doi.org/10.1093/aje/kwm016. PMid:17379619.

MASELLA, R.; SANTANGELO, C.; D'ARCHIVIO, M.; LIVOLTI, G.; GIOVANNINI, C.; GALVANO, F. Protocatechuic acid and human disease prevention: biological activities and molecular mechanisms. Current Medicinal Chemistry, v. 19, n. 18, p. 2901-2917, 2012. http://dx.doi.org/10.2174/092986712800672102. PMid:22519395.

MENEZES, E. M. S.; TORRES, A. T.; SRUR, A. U. S. Valor nutricional da polpa de açaí (Euterpe oleracea Mart) liofilizada. Acta Amazonica, v. 38, n. 2, p. 311-316, 2008. http://dx.doi. org/10.1590/S0044-59672008000200014

MERTENS-TALCOTT, S. U.; RIOS, J.; JILMA-STOHLAWETZ, P.; PACHECO-PALENCIA, L. A.; MEIBOHM, B.; TALCOTT, S. T.; DERENDORF, H. Pharmacokinetics of anthocyanins and antioxidant effects after the consumption of anthocyanin-rich acai juice and pulp (Euterpe oleracea Mart.) in human healthy volunteers. Journal of Agricultural and Food Chemistry, v. 56, n. 17, p. 7796-7802, 2008. http://dx.doi.org/10.1021/jf8007037. PMid: 18693743

MOURA, R. S.; RESENDE, Â. C. Cardiovascular and metabolic effects of açaí, an Amazon plant. Journal of Cardiovascular Pharmacology, v. 68, n. 1, p. 19-26, 2016. http://dx.doi.org/10.1097/ FJC.0000000000000347. PMid:26657713.

OLIVEIRA, P. R. B.; COSTA, C. A.; BEM, G. F.; CORDEIRO, V. S. C.; SANTOS, I. B.; CARVALHO, L. C. R. M.; CONCEIÇÃO, E. P. S.; LISBOA, P. C.; OGNIBENE, D. T.; SOUSA, P. J. C.; MARTINS, G. R.; SILVA, A. J. R.; MOURA, R. S.; RESENDE, A. C. Euterpe oleracea Mart.- derived polyphenols protect mice from diet-induced obesity and fatty liver by regulating hepatic lipogenesis and cholesterol excretion. PLoS One, v. 10, n. 12, p. e0143721, 2015. http://dx.doi.org/10.1371/journal. pone.0143721. PMid:26630290

OLIVEIRA, P. R.; COSTA, C. A.; BEM, G. F.; CAVALHO, L. C.; SOUZA, M. A.; LEMOS NETO, M.; SOUSA, P. J. C.; MOURA, R. S.; RESENDE, A. C. Effects of an extract obtained from fruits of Euterpe oleracea Mart. in the components of metabolic syndrome induced in C57BL/6J mice fed a high-fat diet. Journal of Cardiovascular Pharmacology, v. 56, n. 6, p. 619-626, 2010. http://dx.doi.org/10.1097/FJC.0b013e3181f78da4. PMid:20838232.

PACHECO-PALENCIA, L. A.; DUNCAN, C. E.; TALCOTT, S. T. Phytochemical composition and thermal stability of two commercial açai species, Euterpe oleracea and Euterpe precatoria. Food Chemistry, v. 115, n. 4, p. 1199-1205, 2009. http://dx.doi. org/10.1016/j.foodchem.2009.01.034.

PACHECO-PALENCIA, L. A.; MERTENS-TALCOTT, S.; TALCOTT, S. T. Chemical composition, antioxidant properties, and thermal stability of a phytochemical enriched oil from açai (Euterpe oleracea Mart.). Journal of Agricultural and Food Chemistry, v. 56, n. 12, p. 4631-4636, 2008a. http://dx.doi.org/10.1021/ jf800161u. PMid: 18522407.
PACHECO-PALENCIA, L. A.; TALCOTT, S. T.; SAFE, S.; MERTENSTALCOTT, S. Absorption and biological activity of phytochemical-rich extracts from acai (Euterpe oleracea Mart.) pulp and oil in vitro. Journal of Agricultural and Food Chemistry, v. 56, n. 10, p. 3593-3600, 2008b. http://dx.doi.org/10.1021/jf8001608. PMid: 18442253

POZO-INSFRAN, D.; BRENES, C. H.; TALCOTT, S. T. Phytochemical composition and pigment stability of Acai (Euterpe oleracea Mart.). Journal of Agricultural and Food Chemistry, v. 52, n. 6, p. 1539-1545, 2004. http://dx.doi.org/10.1021/jf035189n. PMid:15030208.

POZO-INSFRAN, D.; PERCIVAL, S. S.; TALCOTT, S. T. Acai (Euterpe oleracea Mart.) polyphenolics in their glycoside and aglycone forms induce apoptosis of $\mathrm{HL}-60$ leukemia cells. Journal of Agricultural and Food Chemistry, v. 54, n. 4, p. 1222-1229, 2006. http://dx.doi.org/10.1021/jf052132n. PMid:16478240.

REIS, C. E. G.; VASCONCELOS, I. A. L.; BARROS, J. F. N. Políticas públicas de nutrição para o controle da obesidade infantil. Revista Paulista de Pediatria, v. 29, n. 4, p. 625-633, 2011. http://dx.doi.org/10.1590/S0103-05822011000400024.

ROCHA, A. P. M.; RESENDE, A. C.; SOUZA, M. A. V.; CARVALHO, L. C. R. M.; SOUSA, P. J. C.; TANO, T.; CRIDDLE, D. N.; PORTO, L. C.; VALENCA, S. S.; MOURA, R. S. Antihypertensive effects and antioxidant action of a hydro-alcoholic extract obtained from fruits of Euterpe oleracea Mart. (Açaí). Journal of Pharmacology and Toxicology, v. 3, n. 6, p. 435-448, 2008. http://dx.doi. org/10.3923/jpt.2008.435.448.

ROCHA, S. M. B. M.; OLIVEIRA, A. G.; COSTA, M. C. D. Benefícios funcionais do açaí na prevenção de doenças cardiovasculares. Journal of Amazon Health Science, v. 1, n. 1, p. 1-10, 2015.

ROGEZ, H.; POMPEU, D. R.; AKWIE, S. N. T.; LARONDELLE, Y. Sigmoidal kinetics of anthocyanin accumulation during fruit ripening: a comparison between açai fruits (Euterpe oleracea) and other anthocyanin-rich fruits. Journal of Food Composition and Analysis, v. 24, n. 6, p. 796-800, 2011. http://dx.doi. org/10.1016/j.jfca.2011.03.015.

RUFINO, M. M. S.; ALVES, R. E.; BRITO, E. S.; PÉREZ-JIMÉNEZ, J.; SAURA-CALIXTO, F.; MANCINI-FILHO, J. Bioactive compounds and antioxidant capacities of 18 non-traditional tropical fruits from Brazil. Food Chemistry, v. 121, n. 4, p. 996-1002, 2010. http://dx.doi.org/10.1016/j.foodchem.2010.01.037.

SCHAUSS, A. G.; WU, X.; PRIOR, R. L.; OU, B.; HUANG, D.; OWENS, J.; AGARWAL, A.; JENSEN, G. S.; HART, A. N.; SHANBROM, E. Antioxidant capacity and other bioactivities of the freeze-dried Amazonian palm berry, Euterpe oleraceae mart. (acai). Journal of Agricultural and Food Chemistry, v. 54, n. 22, p. 8604-8610, 2006. http://dx.doi.org/10.1021/jf0609779. PMid: 17061840

SILVA, L. B.; ANNETTA, F. E.; ALVES, A. B.; QUEIROZ, M. B.; FADINI, A. L.; SILVA, M. G.; EFRAIM, P. Effect of differently processed açai (Euterpe oleracea Mart.) on the retention of 
Propriedades antioxidantes do açaí (Euterpe oleracea) na síndrome metabólica

Cedrim, P. C. A. S. et al.

phenolics and anthocyanins in chewy candies. International Journal of Food Science \& Technology, v. 51, n. 12, p. 26032612, 2016. http://dx.doi.org/10.1111/ijfs. 13245.

SOUZA, M. O.; SILVA, M.; SILVA, M. E.; OLIVEIRA, R. P.; PEDROSA, M. L. Diet supplementation with acai (Euterpe oleracea Mart.) pulp improves biomarkers of oxidative stress and the serum lipid profile in rats. Nutrition, v. 26, n. 7-8, p. 804-810, 2010. http:// dx.doi.org/10.1016/j.nut.2009.09.007. PMid:20022468.

UDANI, J. K.; SINGH, B. B.; SINGH, V. J.; BARRETT, M. L. Effects of Acai (Euterpe oleracea Mart.) berry preparation on metabolic parameters in a healthy overweight population: a pilot study. Nutrition Journal, v. 10, n. 1, p. 45, 2011. http://dx.doi. org/10.1186/1475-2891-10-45. PMid:21569436.

YAMAGUCHI, K. K. L.; PEREIRA, L. F.; LAMARÃO, C. V.; LIMA, E. S.; VEIGA-JUNIOR, V. F. Amazon acai: chemistry and biological activities: a review. Food Chemistry, v. 179, p. 137-151, 2015. http://dx.doi.org/10.1016/j.foodchem.2015.01.055. PMid:25722148.

YUYAMA, L. K. O.; AGUIAR, J. P. L.; SILVA FILHO, D. F.; YUYAMA, K.; VAREJÃO, M. J.; FÁVARO, D. I. T.; VASCONCELLOS, M. B. A.; PIMENTEL, S. A.; CARUSO, M. S. F. Physicochemical characterization of acai juice of Euterpe precatoria Mart. from different amazonian ecosystems. Acta Amazonica, v. 41, n. 4, p. 545-552, 2011. http://dx.doi.org/10.1590/S0044-59672011000400011.

ZAPATA-SUDO, G.; SILVA, J. S.; PEREIRA, S. L.; SOUZA, P. J.; MOURA, R. S.; SUDO, R. T. Oral treatment with Euterpe oleracea Mart.(açaí) extract improves cardiac dysfunction and exercise intolerance in rats subjected to myocardial infarction. BMC Complementary and Alternative Medicine, v. 14, n. 1, p. 227, 2014. http://dx.doi.org/10.1186/1472-6882-14-227. PMid:25000822. 